\title{
Nonlinear Controller with the Enhanced Resistance to Electromagnetic Disturbances
}

\author{
Algirdas Baskys \\ Department of Computer Science and Communications Technologies, \\ Vilnius Gediminas Technical University, \\ Naugarduko St. 41, LT-03227 Vilnius, Lithuania \\ algirdas.baskys@vgtu.lt
}

\begin{abstract}
The controller, which provides the control system dynamics similar to PID controller, but guarantees higher resistance of the control system to electromagnetic disturbances, has been proposed. The controller is similar to Proportional Integral (PI) controller. Distinctive feature of the controller is that the impact of the proportional term on the controller output signal is proportional to the control error raised to a power. The controller investigation results and their comparison with the results obtained using popular PID and PI controllers are presented.
\end{abstract}

Index Terms-Automatic control; Closed loop systems; Nonlinear control systems; Electromagnetic compatibility; Proportional integral control.

\section{INTRODUCTION}

The peculiarity of the controllers that are used for the control of industrial processes is that the controller often has to operate under the impact of electromagnetic disturbances (EMD). The noise signal produced by EMD is usually induced into the bus, which connects the feedback sensor with the controller. The PID (Proportional-IntegralDerivative) controller is commonly employed for the control of industrial processes [1]-[5]. Control systems, based on the PID controller, are featured by good dynamics. It provides a short settling time of system response. However, PID controller is EMD sensitive. Main reason for EMD sensitivity is that controller has the Derivative (D) part [1], [6], [7], which acts as the high pass filter. Because of this, the noise signal produced by EMD, which appears at the controller input, affects the derivative part output and disturbs the operation of the control system. Therefore, the derivative term is often excluded in real applications, i.e., practically, PI controller is applied in place of PID to avoid the control system operation instability [1], [8]-[10]. The PI (Proportional Integral) controller has higher EMD resistance in comparison to the PID controller. However, the system response dynamics is worse when PI controller is applied. Therefore, the settling time of the controlled parameter using PI controller is longer than in the situation when the PID controller is employed.

Modification of the PI controller, which provides the control system dynamics similar to the PID controller, but guarantees higher resistance of the control system to

Manuscript received 11 January, 2018; accepted 25 May, 2019. electromagnetic disturbances, has been presented and analyzed in this work.

\section{CONTROL ALgORITHM}

The control algorithm of the proposed controller is given in (1)

$$
U(t)=K_{\mathrm{P}}|e(t)|^{m} \operatorname{sgn}[e(t)]+K_{\mathrm{I}} \int_{t_{0}}^{t} e(t) \mathrm{d} t,
$$

where $K_{\mathrm{P}}$ and $K_{\mathrm{I}}$ are proportional and integral constants, $U(t)$ is controller output, $t$ is time, $t_{0}$ is initial time moment, $m$ is some real-valued number, and $e(t)$ is control error that acts as the input signal of controller.

The control algorithm (1) is nonlinear and the controller, which is based on it, can be named as a nonlinear PI (nPI) controller. The algorithm (1) presents the ordinary PI controller, when $m=1$.

The impact of the proportional term on the controller output in the nPI controller is proportional to $[e(t)]^{m}$. If $m>1$, this impact is weaker at low $e(t)$ and is stronger when $e(t)$ is high. It means that the nPI controller with $m>1$ tends to the Integral controller if $e(t)$ is low and to the Proportional one if $e(t)$ is high. The integral (I) part acts as the low-pass filter, therefore, the Integral controller has high EMI immunity. Thus, the nPI controller becomes more EMI immune at low $e(t)$ values, i.e., if the system works close to the steady state situation. The impact of the proportional term increases at high $e(t)$ values. This fact speeds up the response of system to set point and plant load changes.

\section{INVESTIGATION PROCEDURE}

The structure of the analyzed feedback control system is presented in Fig.1.

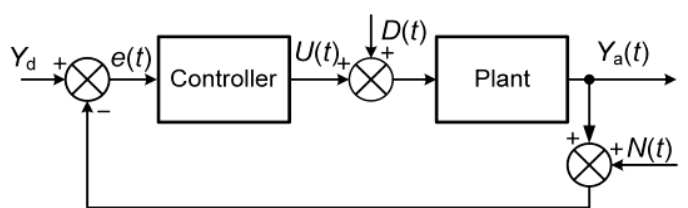

Fig. 1. Feedback control system.

The $Y_{\mathrm{d}}$ is desired (set point) value and $Y_{\mathrm{a}}(t)$ is actual value of the parameter, which is controlled by the system. The system is influenced by the noise signal $N(t)$ produced by EMD. It is 
influenced by the load disturbance $D(t)$ as well.

The Matlab/Simulink was used for the analysis. The investigation was performed for the plants with the following transfer functions that correspond to the dynamics of industrial processes [11], [12]:

$$
\begin{aligned}
& G_{1}(s)=\frac{\mathrm{e}^{-3 s}}{(s+1)}, \\
& G_{2}(s)=\frac{1}{(s+1)^{3}} .
\end{aligned}
$$

The transfer function (2) presents the dead time plant and function (3) - the plant with the dynamics described by the third-order transfer function. The saturation block with the +2 upper and -2 lower saturation values was included into the plant models as well.

The dynamic performance of systems with the nPI controller has been investigated for the cases: system is not influenced by EMD, i.e., the noise signal $N(t)=0$; system is influenced by Band-Limited White noise signal. The investigation results have been compared with these obtained using PID and PI controllers. The robustness of the systems, based on the nPI controller, was investigated under uncertainty of the plant parameters as well.

The controllers were tuned using the Nonlinear Control Design (NCD) Blockset of software Simulink for the minimal settling time of set point step response with overshoot not higher than $5 \%$. The parameters of controllers for control systems of plant $G_{1}(s)$ and plant $G_{2}(s)$ are given in Table I.

\begin{tabular}{|c|c|c|c|}
\hline Plant Controller & nPI & PI & PID \\
\hline$G_{1}(s)$ & $\begin{array}{c}K_{\mathrm{p}}=0.57 \\
K_{\mathrm{i}}=0.226 \\
m=3\end{array}$ & $\begin{array}{c}K_{\mathrm{p}}=0.38 \\
K_{\mathrm{i}}=0.205\end{array}$ & $\begin{aligned} K_{\mathrm{p}} & =0.53 \\
K_{\mathrm{i}} & =0.23 \\
K_{\mathrm{d}} & =0.30\end{aligned}$ \\
\hline$G_{2}(s)$ & $\begin{array}{c}K_{\mathrm{p}}=1.64 \\
K_{\mathrm{i}}=0.51 \\
m=1.84\end{array}$ & $\begin{array}{c}K_{\mathrm{p}}=0.90 \\
K_{\mathrm{i}}=0.405\end{array}$ & $\begin{array}{c}K_{\mathrm{p}}=3,20 \\
K_{\mathrm{i}}=0.59 \\
K_{\mathrm{d}}=2.00\end{array}$ \\
\hline
\end{tabular}

TABLE I. CONTROLLER PARAMETERS.

\section{INVESTIGATION RESULTS}

The set point and load disturbance unit step responses and dynamic parameters of systems with plant $G_{1}(s)$, and plant $G_{2}(s)$ using the nPI, PI, and PID controllers are presented in Fig. 2 and Table II. The $t_{\mathrm{s}}$ in Table II is the settling time of the set point step response, $t_{\mathrm{d}}$ is the settling time of the load disturbance step response, and $M_{\mathrm{d}}$ is the overshoot caused by the load disturbance. The values of $t_{\mathrm{s}}$ and $t_{\mathrm{d}}$ are estimated for the $\pm 5 \%$ tolerance band of the controlled parameter $Y_{\mathrm{a}}(t)$.

The obtained results, which are given in Fig. 2 and Table II, show that nPI controller provides shorter $t_{\mathrm{s}}$ and $t_{\mathrm{d}}$ compared to the case when PI controller has been applied. The employment of the nPI controller in the case of plant $G_{1}(\mathrm{~s})$, which has the response delay, allows us to achieve shorter $t_{\mathrm{s}}$ as compared with PID controller. The nPI controller provides a shorter $t_{\mathrm{d}}$ of analyzed systems compared to that of the PID and PI controllers. However, the $M_{\mathrm{d}}$ of systems with the nPI controller for both analyzed plants is higher in comparison with the situation when the
PID controller has been applied.

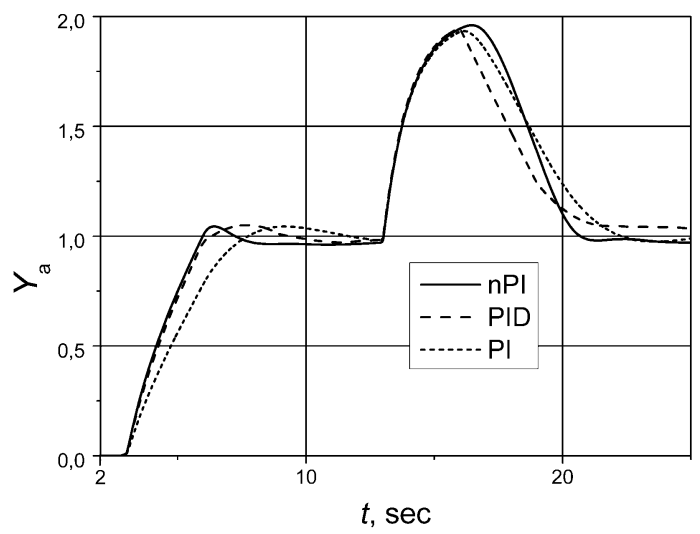

(a)

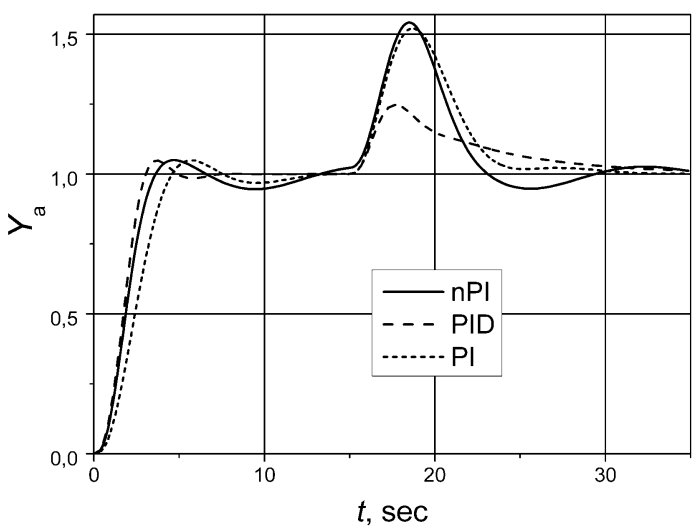

(b)

Fig. 2. The set point and load disturbance unit step responses of systems with plants $G_{1}(s)$ (a) and $G_{2}(s)$ (b) using nPI, PID, and PI controllers.

TABLE II. DYNAMIC PARAMETERS OF THE CONTROL SYSTEM.
\begin{tabular}{|c|c|c|c|}
\hline Controller & nPI & PI & PID \\
\hline \multirow{3}{*}{$\boldsymbol{G}_{\mathbf{1}}(\boldsymbol{s})$} & $t_{\mathrm{s}}=5.7$ & $t_{\mathrm{s}}=7.1$ & $t_{\mathrm{s}}=5.9$ \\
& $t_{\mathrm{d}}=7.2$ & $t_{\mathrm{d}}=8.5$ & $t_{\mathrm{d}}=8.5$ \\
& $M_{\mathrm{d}}=0.96$ & $M_{\mathrm{d}}=0.94$ & $M_{\mathrm{d}}=0.94$ \\
\hline & $t_{\mathrm{s}}=3.2$ & $t_{\mathrm{s}}=4.2$ & $t_{\mathrm{s}}=2.8$ \\
$\boldsymbol{G}_{\mathbf{2}}(\boldsymbol{s})$ & $t_{\mathrm{d}}=7.3$ & $t_{\mathrm{d}}=8.4$ & $t_{\mathrm{d}}=11.6$ \\
& $M_{\mathrm{d}}=0.54$ & $M_{\mathrm{d}}=0.52$ & $M_{\mathrm{d}}=0.25$ \\
\hline
\end{tabular}

The impact of noise signal $N(t)$ on the operation of systems with plant $G_{1}(s)$ and plant $\mathrm{G}_{2}(s)$ using nPI and PID controllers has been investigated. The Band-Limited White noise with the sample time of $0.01 \mathrm{~s}$ and seed [23341] was used Fig. 3(a). The first order low-pass filter was employed for the filtering of noise signal to make the signal similar to actual noise signals. The transfer function of the filter is $G_{\mathrm{f}}(s)=1.2 /(0.004 s+1)$. The filtered signal is given in Fig. 3(b).

The set point step responses of systems influenced by noise signal were investigated. Firstly, the control system was analyzed at the low amplitude of the noise signal, at which the impact of the noise on the controlled parameter of system with PID controller becomes evident. The obtained transients (see Fig. 4) show that the noise signal with the amplitude $A_{N}=0.02-0.03$ (noise signal power $(1-2) \times 10^{-6}$ ) causes the ripples with the amplitude up to 0.05 . However, the noise signal with the same amplitude does not influence the response of the systems in the situation when they are 
based on the nPI controller.

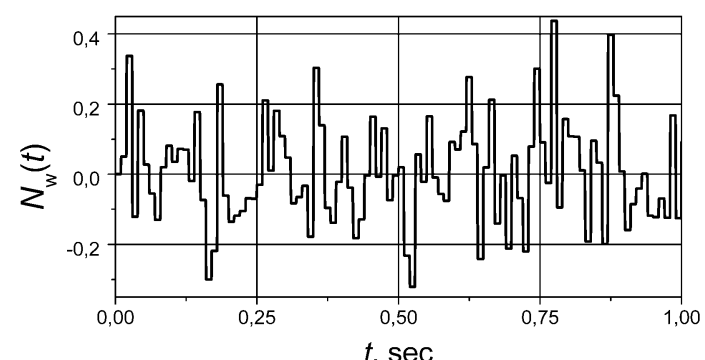

(a)

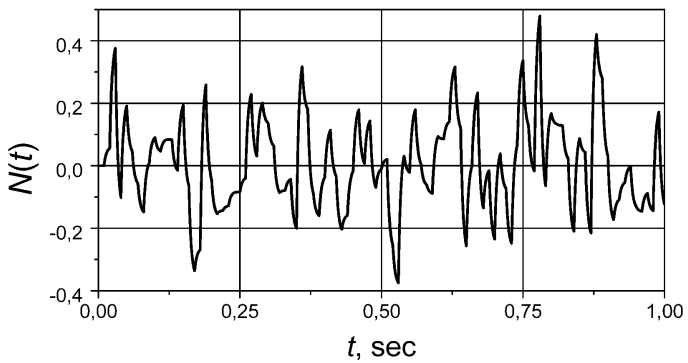

(b)

Fig. 3. The Band-Limited White noise with the sample time of $0.01 \mathrm{~s}$, seed [23341], and power $2 \times 10^{-4}$ (a) and filtered Band-Limited White noise (b).

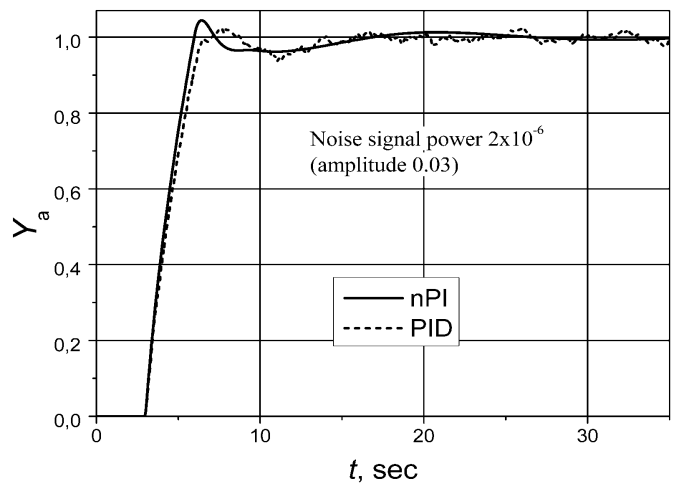

(a)

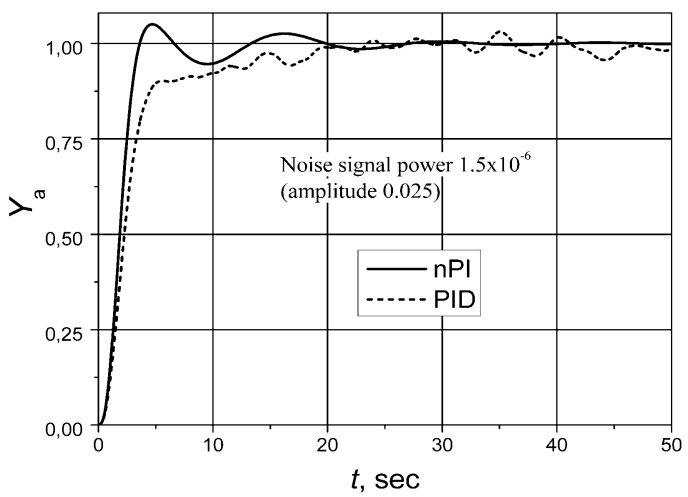

(b)

Fig. 4. The set point unit step response of systems with plants (a) $G_{1}(s)$ and (b) $G_{2}(s)$ using nPI and PID controllers affected by the noise signal with a low amplitude.

The control systems were investigated at high amplitudes of the noise signal $A_{\mathrm{N}}=0.4-0.7$ (noise signal power (2-7)x $10^{-}$ ${ }^{4}$ ), at which the controlled parameter ripples of systems with nPI controller reach value 0.05 . The set point step responses of systems influenced by high amplitude noise are given in Fig. 5. They show that operation of systems using PID controller changes qualitatively - the response of systems becomes slow with large ripples.
The first order low-pass filter can be used with derivative term of the PID controller for reduction of noise impact [6][10]. The transfer function of the filter has to be as follows: $G_{\mathrm{LPF}}(s)=1 /\left[\left(0.1 s K_{\mathrm{d}} / K_{\mathrm{p}}\right)+1\right] \quad[6], \quad[13]$. The responses of systems with PID controller using low-pass filter for the derivative term at the high amplitude of the noise signal are presented in Fig. 5. They show that the filter reduces ripples of the controlled parameter and speeds up the response compared to the situation when PID controller without the filter has been employed. However, the responses of systems are slower and ripples are higher in comparison to systems with nPI controller. It is necessary to stress that the application of the low-pass filter reduces the performance and the robustness of systems with PID controllers [5], [9], [12], [13].

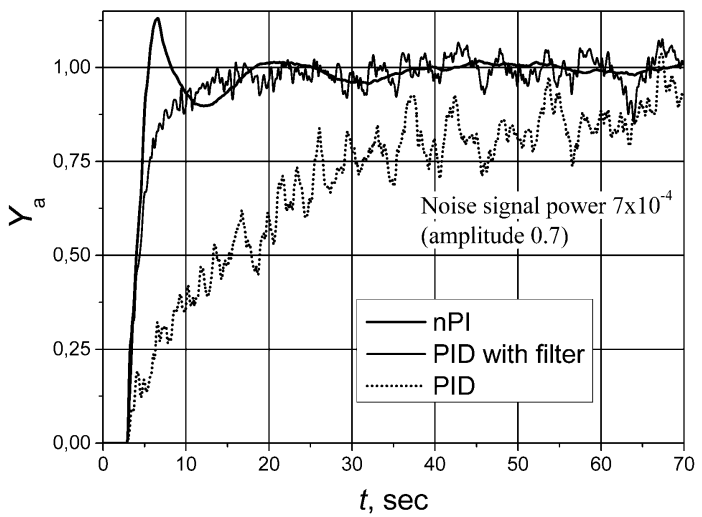

(a)

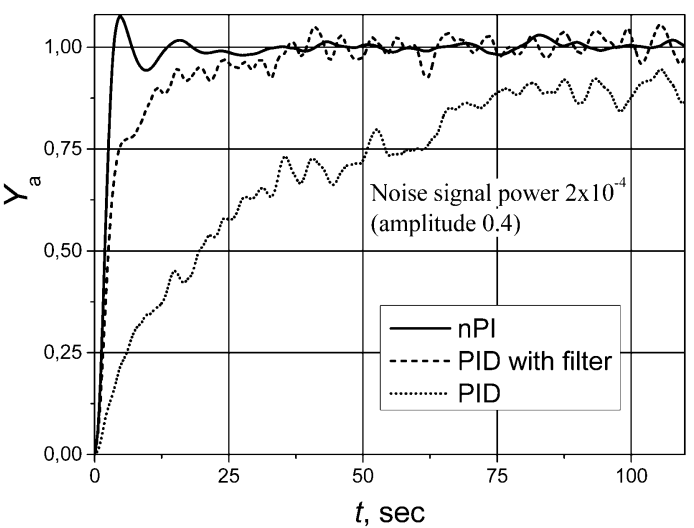

(b)

Fig. 5. The set point unit step response of systems with plants $G_{1}(s)$ (a) and $G_{2}(s)$ (b) using nPI, PID controllers and PID controller with low-pass filter affected by the noise signal with a high amplitude.

It is very relevant to know the response of systems to the uncertainty of plant dynamic parameters [14], [15]. The set point and load disturbance unit step responses of systems with plant $G_{1}(s)$ (a) and plant $G_{2}(s)$ (b) using nPI controller for the situations when the plant dynamic parameters (plant response delay and time constant) decrease simultaneously by $50 \%$ and increase simultaneously by $10 \%, 30 \%$, and $50 \%$ was analyzed. The results are presented in Fig. 6 . They show that systems with both analyzed plants remain stable, just the transition durations and oscillation amplitudes increase.

The upper bounds of plant parameters values, above which the control systems become unstable, were estimated. The system with the plant $G_{1}(s)$ starts operate not stably if the plant response delay and time constant increase 
simultaneously more than by $120 \%$ and the system with the plant $\mathrm{G}_{2}(s)$ becomes not stable if the plant time constant rises more than by $190 \%$.

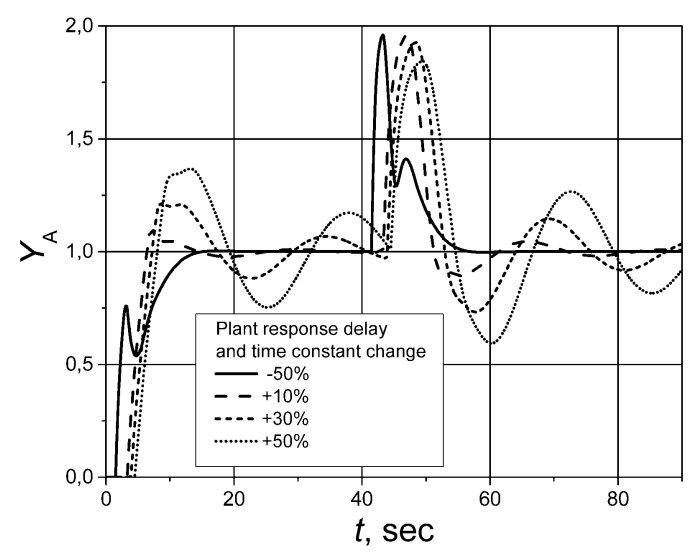

(a)

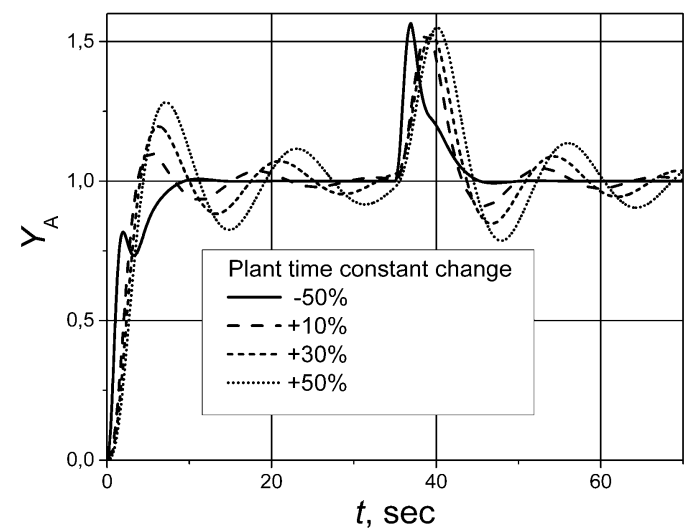

(b)

Fig. 6. The set point and load disturbance unit step responses of systems with plants $G_{1}(s)$ (a) and $G_{2}(s)$ (b) using nPI controller under uncertainty of plant dynamic parameters.

\section{CONCLUSIONS}

The proposed nPI controller provides the dynamics of the control systems similar to that of the PID controller. However, it guarantees a much higher EMD resistance of systems as the PID controller provides.

The control systems based on the nPI controller operate with analyzed plants without substantial changes of the dynamic performance when the amplitude of the noise signal is $A_{\mathrm{N}}<0.4-0.7$. The work of systems with the PID controller at the same noise signal amplitudes changes qualitatively the systems response becomes slow with large ripples.

The low-pass filter, which is used with the derivative term of PID controller, reduces ripples of the controlled parameter and speeds up the response of systems influenced by noise signal. However, the response is slower and ripples are higher as compared to systems with nPI controller.

The system with the nPI controller and plant $G_{1}(s)$ remains stable if the plant response delay and time constant increase simultaneously not more than by $120 \%$ and the system with the plant $\mathrm{G}_{2}(s)$ is stable if the plant time constant rises not more than by $190 \%$.

\section{REFERENCES}

[1] P. O. Larsson and T. Hägglund, "Control signal constraints and filter order selection for PI and PID controllers", in Proc. American Control Conference, San Francisco, CA, USA, pp. 4994-4999, 2011. DOI: 10.1109/ACC.2011.5991112.

[2] T. L. Blevins, "PID Advances in Industrial Control", in Proc. IFAC Conference on Advances in PID Control (PID'12), Brescia, Italy, pp.1-6, 2012. DOI: 10.3182/20120328-3-IT-3014.00004

[3] A. Visioli, "Research Trends for PID Controllers", Acta Polytechnica, vol. 52 no. 5, pp.144-150, 2012.

[4] E. Sariyildiz, H. Yu, and K. Ohnishi, "A Practical Tuning Method for the Robust PID Controller with Velocity Feed-Back", Machines, no3, pp. 208-222, 2015. DOI: 10.3390/machines3030208.

[5] S. Srivastava and V. S. Pandit, "A PI/PID controller for time delay systems with desired closed loop time response and guaranteed gain and phase margins", Journal of Process Control, vol. 37, pp. 70-77, 2016. DOI: $10.1016 /$ j.jprocont.2015.11.001.

[6] A. J. lsaksson and S. F. Graebe, "Derivative filter is an integral part of PID design", IEE Proc.-Control Theory Appl., no. I, pp. 41-45, 2002. DOI: 10.1049/ip-cta:2002011.

[7] T. Hagglund, "Signal Filtering in PID Control", in Proc. IFAC Conference on Advances in PID Control (PID'12), Brescia, Italy, pp. 1-10, 2012. DOI: 10.3182/20120328-3-IT-3014.00002.

[8] T. B. Sekara and M. R. Matausek, "Optimization of PID Controller Based on Maximization of the Proportional Gain Under Constraints on Robustness and Sensitivity to Measurement Noise", IEEE Transactions on Automatic Control, vol. 54, no. 1, pp. 184-189, 2009. DOI: $10.1109 /$ TAC.2008.2008359.

[9] O. Garpinger and T. Hagglund, "Software-based optimal PID design with robustness and noise sensitivity constraints", Journal of Process Control, vol. 33, pp. 90-101, 2015. DOI: 10.1016/j.jprocont.2015.06. 001.

[10] V. M. Alfaro and R. Vilanova, "Performance and Robustness Considerations for Tuning of Proportional Integral/Proportional Integral Derivative Controllers with Two Input Filters", Industrial \& Engineering Chemistry Research, vol.52, pp. 18287-18302, 2013, DOI: dx.doi.org/10.1021/ie4012694.

[11] B. Kristiansson and B. Lennartson, "Robust and optimal tuning of PI and PID controller", IEE Proc.-Control Theory, Appl., vol. 149, no. 1, pp. 17-25, 2002. DOI: 10.1049/ip-cta:20020088.

[12] O. Garpinger, T. Hagglund, and K. J. Astrom, "Performance and robustness trade-offs in PID control", Journal of Process Control, vol. 24, no. 5, pp. 568-577, 2014. DOI: 10.1016/j.jprocont.2014.02. 020 .

[13] H. Panagopoulos, K.J.Astrom, and T.Hagglund, "Design of PID controllers based on constrained optimization", IEE Proc.-Control Theory, Appl., vol. 149, no. 1, pp. 32-40, 2002. DOI: 10.1049/ipcta:20020102.

[14] Q. Wang and R. F. Stengel, "Robust control of nonlinear systems with parametric uncertainty", Automatica, vol. 38, pp. 1591-1599, 2002. DOI: $10.1016 / \mathrm{S} 0005-1098(02) 00046-8$.

[15] J. Závacká, M. Bakošová, and K. Vaneková, "Control of systems with parametric uncertainties using a robust PI controller", $A T \& P$ journal PLUS2, Robust and Adaptive Control, pp. 84-87, 2007. 